tigten Forderung, möglichst bald eine Konferenz zur Anpassung der Seekriegsregeln an das weiter entwickelte humanitäre Völkerrecht einzuberufen. Das von ihm in Anlehnung an "UNCLOS" für die Seerechtskonferenz auf Grund der englischen Konferenzbezeichnung "United Nations Conference on the Law of Armed Conflict at Sea" vorgeschlagene Akronym lautet UNCLACS.

Hierzu kein weiterer Kommentar.

Karl Leuteritz

\title{
Donat Pharand
}

\section{Northwest Passage: Arctic Straits}

In Association with Leonard H. Legault, Martinus Nijhoff Publishers, Dordrecht, 1984, XXII, 199 S.

Der Kanadier Donat Pharand ist Autor einer Vielzahl rechtswissenschaftlicher Untersuchungen über die Arktis, von denen hier nur das Buch "Law of the Sea of the Arctic with Special Reference to Canada" (1973) und die Haager Vorlesung "The Legal Status of the Artic Regions" (RdC 1979 II, Bd. 163, S. 48 bis 115) erwähnt seien. Die vorliegende Studie ist in der Reihe "International Straits of the World" erschienen, die Gerard Mangone seit 1978 herausgibt. ${ }^{1}$ Ob die Nordwest-Passage freilich überhaupt eine "Meerenge" im Rechtssinne darstellt (und welchen Typ einer Meerenge sie gegebenenfalls verkörpert) ist eine Frage, die näherer Uberlegung bedarf. Die Antwort hierauf wie auch die Beurteilung der weiteren, mit dem völkerrechtlichen Status der Nordwest-Passage wie auch überhaupt der an Kanada grenzenden Teile der Arktis sind Probleme von außerordentlicher wirtschaftlicher und strategischer Bedeutung. Beide Umstände beruhen auf neueren Entwicklungen: In den siebziger Jahren haben die Explorationen im arktischen Festlandsockel, die schon in früherer Zeit begonnen worden waren, Gewißheit über reiche Ol- und Erdgasvorkommen geschaffen, deren Ausbeutung allerdings erhebliche technische und Transportschwierigkeiten aufwirft; seit der Fahrt der "Nautilus" (1958) ist das von Packeis überzogene arktische Meer zudem Operationsgebiet von Unterseebooten - in einem Gebiet, das zwischen sowjetischen und amerikanischen Marinestützpunkten liegt und für andere Verkehrsmittel weitgehend unzugänglich ist, von besonderer Bedeutung.

Pharands Buch ist in 11 Kapitel gegliedert und wegen seiner vorbildlichen Ubersichtlichkeit, seinen reichhaltigen, genauen Karten und Tabellen sowie der klaren Sprache gleichermaßen als Nachschlagewerk wie zur zusammenhängenden Lektüre geeignet. Der Autor beginnt mit einer Beschreibung der (sechs unterschiedlichen) Routen, auf denen

1 Vgl. die Rezension von U. Jenisch zu Gunnar Alexanderssons The Baltic Straits, 1982, in: VRU 17 (1984), S. 398 f.; die Parallelstudie zur Nordost-Passage ist schon 1978 erschienen: W. E. Butler, Northeast Arctic Passage. 
die "Nordwest Passage" möglich ist; nur zwei sind für größere Schiffseinheiten geeignet. Zwei weitere Kapitel schildern detailliert die zunächst unter britischer, seit 1880 vorwiegend unter kanadischer Ägide durchgeführten Expeditionen in dem Gebiet. Zwischen 1903 und 1983 hat es nur 40 volle Durchquerungen der Passage gegeben. Bereits Mitte des 19. Jahrhunderts waren aber die meisten Inseln des kanadischen Archipels in der Arktis erforscht worden. Nach dem 2. Weltkrieg hat Kanada insbesondere Eisbrecher der Küstenwacht, Forschungsschiffe und Marine-Fahrzeuge in die Region entsandt.

Kapitel 4 gibt einen kurzen Ưberblick über die Entwicklung der ökonomischen Bedeutung der Nordwest-Passage und über die zum Einsatz kommenden Bohrgeräte, die den widrigen Förderungsbedingungen angepaßt sein müssen - insoweit ist für die unmittelbare Zukunft mit einem technologischen Standard zu rechnen, der eine Förderung in großer Kapazität ermöglicht. Im Jahr 1982 wurden im Gesamtgebiet des Festlandsokkelabhanges von Alaska und den Schelfgebieten der Beaufort-See ca. 1,5 Mill. Barrel Öl pro Tag gefördert. Allein in dem einzigen bisher erschlossenen Feld in der Prudhoe Bay sind aber über 7 Milliarden Barrel vorhanden.

Der Autor schildert sodann die anfallenden Transportprobleme und diskutiert zahlreiche Tanker- und Rohrleitungstypen. Seiner Einschätzung nach wird zu Beginn der 1990er Jahre das technische Potential vorhanden sein, das für die reibungslose Verbringung der dann zu erwartenden Fördermenge in außerarktische Gebiete erforderlich ist ob jenes Datum wirklich schon den Beginn großangelegter Fördertätigkeit markieren wird, ist allerdings nach Abschluß des Buches infolge der jüngeren Olpreisentwicklung eher fragwürdig geworden (worauf der Autor im Vorwort hinweist).

Die Erwartung einer drastischen Zunahme kommerzieller Aktivität in den arktischen Gebieten bildet den Anlaß zu der nun ansetzenden völkerrechtlichen Analyse des Autors, die die zweite Hälfte des Buches ausmacht. Nach einer Skizze der Aussagen des Völkerrechts zum Meerengenproblem umreißt Pharand den gegenwärtigen Status der Nordwest-Passage. Bekanntlich sind in dem UNO-Seerechtsübereinkommen von 1982 nur Meerengen gesondert geregelt, die der internationalen Schiffahrt dienen, während andere Meerengen dem seerechtlichen Regime des jeweiligen Meeresgebietes unterfallen. Die Nordwest-Passage stellt (wie die Nordost-Passage) zweif elsf rei eine "Meerenge" dar; ob sie auch eine "internationale" Meerenge ist, hängt davon ab, ob sie auch der "internationalen Schiffahrt" dient. Nur dann käme das Regime der sog. Transit-Passage zur Anwendung, das dem Küstenstaat geringere Hoheitsrechte einräumt als das Regime der sog. Innocent Passage, wie es im allgemeinen im Küstenmeer Anwendung findet. Bei Anwendbarkeit der Regeln über die Transit-Passage wären beispielsweise das Recht des Uberflugs und das Durchfahrtsrecht für in getauchtem Zustand fahrende Unterseeboote gewährleistet. Die bisherige geringe Durchfahrtsquote (nur 11 Schiffe unter nicht-kanadischer Flagge in 80 Jahren), der experimentelle Charakter der Passagen und der Um-

2 Vgl. nur R. Wolfrum, Die Beschränkungen für die Freiheit der Schiffahrt durch das kanadische "Arctic Waters Pollution Prevention Act " sowie die internationalen Ubereinkommen zum Schutze der Meeresumwelt, in: R. Bernhardt/W. Rudolf (Hrsg.), Die Schiffahrtsf reiheit im gegenwärtigen Völkerrecht, 1975, S. 143 ff.; U. Jenisch, Der Arktische Ozean und das Neue Seerecht, in: Außenpolitik 35 (1984), S. 204 ff. 
stand, daß Kanada für sich das Recht der Zustimmung im Einzelfall in Anspruch genommen hat, lassen Pharand die "Internationalität" der Nord-West-Passage verneinen; die bloße Möglichkeit eines intensiveren internationalen Schiffsverkehrs reiche nicht aus. Die dieser Frage gewidmeten Teile des Buches können hier nicht im einzelnen diskutiert werden; sie lassen die eingehende Auseinandersetzung mit der Konferenzgeschichte der 3. Seerechtskonferenz und mit literarischen Stimmen hierzu etwas vermissen und stützen sich allzu sehr auf die Entscheidung des Internationalen Gerichtshofs im KorfuKanal-Fall von 1949.

Konsequent für seinen Ausgangspunkt erörtert Pharand sodann die Einzelheiten des Rechts der friedlichen Durchfahrt, insbesondere auch die Durchfahrtsrechte für Kriegsschiffe, ehe er sich den Möglichkeiten Kanadas zuwendet, Maßnahmen zum Schutz der Nordwest-Passage zu ergreifen. Im Mittelpunkt steht dabei der "Artic Waters Pollution Prevention Act " von 1970, der auf kräftigen Widerstand der Vereinigten Staaten gestoßen war, sowie - als aktuelle völkerrechtliche Rechtsgrundlage - der speziell eisbedeckten Gebiete gewidmete Art. 234 der Seerechts-Konvention von 1982.

Ein rechtspolitischer Ausblick diskutiert eventuelle weitere Eingriffsmöglichkeiten Kanadas, die über dessen bisherige Praxis hinausgehen würden und widmet sich insbesondere der Frage, durch welche Entwicklungen die Nordwest-Passage den vom Autor bisher abgelehnten Status einer Meerenge, für die die Regeln der Transit-Passage gelten würden, erlangen könnte. Da Pharand - wie erwähnt - insoweit vor allem auf den Umfang der internationalen Schiffahrt abstellt, sieht er angesichts der zu erwartenden Zunahme kommerziell veranlaßter Schiffahrtsaktivität in der Arktis die Wahrscheinlichkeit, daß der Status der Nordwest-Passage zu einem nicht allzu fernen Zeitpunkt umschlägt, sofern nicht Kanada dem mit den derzeit zu Gebote stehenden rechtlichen Möglichkeiten entgegentritt.

Die drei abschließenden Kapitel sind den ökologischen und sicherheitspolitischen Fragen der Arktis vor dem Hintergrund der zuvor beschriebenen Rechtslage, ferner den Auswirkungen auf die Lebensweise der Eskimo-Bevölkerung (Inuit) gewidmet. Eine wesentliche Verschlechterung des ökologischen Gleichgewichts in der Region hält Pharand nur dann für vermeidbar, wenn Kanada von den derzeit vorhandenen (sich möglicherweise künftig verringernden) rechtlichen Eingriffsmöglichkeiten in weiterem Umfang als bisher Gebrauch machte. Zur Erhaltung des historisch gewachsenen Status Quo der Eskimo-Bevölkerung sei eine Klärung derzeit umstrittener Statusrechte aufgrund innerstaatlichen kanadischen Rechts nötig, das die Besiedelung bewohnbarer Teile der Arktis fördere, um auf diese Weise Kanadas völkerrechtliche Position zu stärken.

Das Buch wird vervollständigt durch eine nützliche Auswahlbibliographie, die - soll man sagen: wie gewöhnlich? - deutschsprachige Arbeiten nicht zur Kenntnis nimmt. Es bietet eine gute Grundlage für die Beschäftigung mit den Rechtsfragen eines ökonomisch, sicherheitspolitisch und ökologisch hochinteressanten Raumes, der zu den letzten verbleibenden Gebieten der Erde gehört, in denen nationalstaatliche Ansprüche auf solche stoßen, die vom Gedanken eines "Common-Heritage-Prinzips" angeleitet sind. Wie Pharand die historischen und tatsächlichen Hintergründe, auch die Konsequenzen faktischer 
Veränderungen, aufhellt und in die rechtliche Analyse einbezieht, ist vorbildlich; wie er den kanadischen Interessenstandpunkt verdeutlicht und die Möglichkeiten seiner rechtlichen Umsetzung diskutiert, ist exemplarisch - ein hochinteressantes, eingehende Lektüre lohnendes Buch.

Philip Kunig

\section{Michel Doucet}

\section{Wörterbuch der Rechts- und Wirtschaftssprache}

begründet von Michel Doucet, fortgeführt von Klaus W. Fleck, Teil II, Deutsch-Französisch, 4. erweiterte Auflage, München: C. H. Beck, 1985, pp xvi, 656, DM 108,-(Leinen)

Der neu erschienene deutsch-französische Teil des bewährten Werks ist im Umfang erheblich erweitert und bietet nun auch einen Anhang deutschsprachiger Abkürzungen mit deren Langform und französischer Ubertragung. Der Band umfaßt in seinen ca. 38000 Stichwörtern zahlreiche Fachtermini auch entlegener Sachgebiete. Der ausgewählte Wortschatz fußt weitgehend auf dem von Creifelds Rechtswörterbuch, anhand dessen der sachliche Zusammenhang der Einträge weiter bestimmt werden kann.

Regionale sprachliche Besonderheiten innerhalb der deutschen und französischen Sprachgebiete werden gekennzeichnet, ebenso wie zum Teil der Sprachgebrauch im Rahmen der Europäischen Gemeinschaften; Ausdrücke, die Begriffe benennen, welche in den Rechts- und Wirtschaftsordnungen der Ausgangs- bzw. Zielsprachgebiete kein Gegenstück haben, werden neben der angegebenen Ubersetzung zusätzlich durch Fußnoten erläutert.

Unbeschadet des oft vergessenen Umstands, auf den der Neubearbeiter zu Recht hinweist, daß eine genaue semantische Entsprechung sich oft erst aus konkreten Zusammenhängen ergibt, die kein Wörterbuch ganz erfassen kann, wird diese Neuauflage dem praxisorientierten Benutzer zahlreiche Sprachprobleme schnell und zufriedenstellend lösen helfen. 